
allemande

49-1 | 2017

Berlin 1957-1994

\title{
Les jumelages économiques franco-allemands. Le cas des chambres de commerce et d'industrie de Colmar et Fribourg-en-Brisgau
}

Martial Libera

\section{(2) OpenEdition Journals}

Édition électronique

URL : https://journals.openedition.org/allemagne/539

DOI : 10.4000/allemagne.539

ISSN : 2605-7913

Éditeur

Société d'études allemandes

Édition imprimée

Date de publication : 16 juin 2017

Pagination : 209-222

ISSN : 0035-0974

\section{Référence électronique}

Martial Libera, «Les jumelages économiques franco-allemands. Le cas des chambres de commerce et d'industrie de Colmar et Fribourg-en-Brisgau », Revue d'Allemagne et des pays de langue allemande [En ligne], 49-1 | 2017, mis en ligne le 16 juin 2018, consulté le 03 juin 2022. URL: http://

journals.openedition.org/allemagne/539; DOI : https://doi.org/10.4000/allemagne.539 


\title{
Les jumelages économiques franco-allemands. Le cas des chambres de commerce et d'industrie de Colmar et Fribourg-en-Brisgau
}

\author{
- Martial Libera*
}

L'histoire des jumelages franco-allemands après 1945 est aujourd'hui bien connue. Les recherches entreprises sur ce sujet se sont concentrées sur les appariements ${ }^{(1)}$ entre villes ou collectivités territoriales ${ }^{(2)}$. Ces travaux ont montré que la réconciliation franco-allemande n'aurait pu aboutir sans les rapprochements menés à la base, entre acteurs de la société civile. C'est qu'en effet par leur ampleur, par leur précocité également, la dynamique de ces relations transnationales, très largement indépendantes des États, a "permis d'ancrer la coopération franco-allemande au niveau local et régional et d'impliquer une très grande partie des deux sociétés dans l'échange bilatéral grâce à un maillage étroit des deux territoires» ${ }^{(3)}$. En définitive, ces jumelages ont constitué un préalable nécessaire au rapprochement durable entre les gouvernements français et allemand et, plus globalement, à la réconciliation franco-allemande ${ }^{(4)}$. Jusqu'aux

* Maître de conférences HDR en histoire contemporaine à l'Université de Strasbourg, DynamE (UMR 7367).

1 Avant 1945 , le terme «appariement» est utilisé. Celui de jumelage n’apparaît qu’après la Deuxième Guerre mondiale.

2 Sur les jumelages entre villes, voir, par exemple, l'étude pionnière de John E. FARQUHARson et Stephen C. Holt, Europe from Below: An assesment of Franco-German Popular Contacts, New York, St. Martin's press, 1975 et, pour les jumelages des lendemains de guerre, Corine Defrance, «Les premiers jumelages franco-allemands, 1950-1963», Lendemains, nº 21 (1996), p. 83-95. Sur les jumelages entre régions, voir, à titre d'exemple, Christian SebeKe, «"Wir müssten Brücken bauen von Mensch zu Mensch”. Die Partnerschaft Rheinland-Pfalz - Burgund als regionales Annäherungsmodell», in: Corine DefrAnCE, Michael Kißener et Pia Nordblom (dir.), Wege der Verständigung zwischen Deutschen und Franzosen nach 1945. Zivilgesellschaftliche Annäherungen, Tübingen, Narr Verlag, 2010, p. 311-326.

3 Corine Defrance, «Les jumelages franco-allemands. Aspects d'une coopération transnationale», Vingtième Siècle. Revue d'histoire, no 99 (2008), p. 189-201, ici p. 199.

4 Corine Defrance (dir.), "La "réconciliation" après les conflits: un "savoir-faire" européen?", Les Cahiers Sirice, $\mathrm{n}^{\circ} 15$ (2016). Voir aussi dans Nicolae Paun, Sylvain Schirmann (éd.), Borders, 
années 1960 et 1970, les activités des jumelages entre villes et collectivités territoriales ont été centrées sur des domaines relevant avant tout des sphères culturelles et éducatives, comme la formation de la jeunesse, la culture et la science, la presse et la radio, le sport. À partir des années 1970 et 1980, et plus encore dans les années 1990, la dimension économique des jumelages s'affirme et tend même à prévaloir sur les questions d'ordre culturel ${ }^{(5)}$.

Ce tropisme progressif vers l'économique conduit à interroger l'existence de jumelages franco-allemands à vocation strictement économique. Dans ce domaine, les chambres de commerce et d'industrie (CCI) portent l'essentiel des initiatives ${ }^{(6)}$. Aujourd'hui, ceux-ci font d'ailleurs partie de leurs pratiques courantes ${ }^{(7)}$. Mais tel n'était pas le cas au sortir de la Deuxième Guerre mondiale. Sur l'espace franco-allemand, il faut attendre 1959 pour que deux compagnies consulaires, celle de Colmar, dans le Haut-Rhin, et celle de Fribourg-en-Brisgau, dans le Bade, décident de se jumeler. L'histoire de ce jumelage économique peut s'analyser selon plusieurs perspectives complémentaires. Au-delà du rôle déterminant des acteurs qui ont œuvré à sa réalisation, ce jumelage met en lumière le poids des forces profondes, qu'il s'agisse de l'ancienneté des relations commerciales existant de part et d'autre du Rhin ou de la complémentarité et de l'interdépendance des économies alsacienne et badoise. Il est également le fruit de contraintes extérieures, avec la nécessité pour ces régions frontalières de s'adapter à la nouvelle donne européenne induite par le Marché commun. Le jumelage conduit par ailleurs à repenser les relations entre État et acteurs économiques parce qu'il permet aux acteurs consulaires de s'affranchir en partie de la tutelle publique et d'initier leurs «propres » relations bilatérales. Sur le plan francoallemand et transfrontalier, ce jumelage montre enfin l'antériorité d'initiatives privées sur celles des États et le rôle d'incubateur qu'elles ont joué dans la réconciliation des deux pays.

Pour comprendre la genèse du jumelage, les relations entre chambres de commerce françaises et allemandes seront abordées depuis la fin de la guerre. C'est qu'en effet, de 1945 à 1959, le contexte est à maints égards favorable au rapprochement entre les compagnies alsaciennes et badoises. Dans un deuxième temps, il s'agira d'explorer l'âge d'or du jumelage dans les années 1960. Un troisième mouvement analysera la montée de diverses difficultés qui, à partir de la crise de 1973, conduisent à une mise en sommeil progressive du jumelage puis, au début des années 1980, à sa réactivation.

Identities, Communities. The Road to Reconciliation and Partnership in Central and Eastern Europe, Baden-Baden, Nomos, 2016, la partie dirigée par Sylvain Schirmann sur «la réconciliation francoallemande, un modèle» (p. 209-334).

5 C. Defrance, «Les jumelages franco-allemands» (note 3), p. 198.

6 Rappelons ici que, dans les deux pays, les compagnies consulaires, organismes parapublics, sont chargées de représenter auprès de leurs pouvoirs publics les intérêts des industriels et des commerçants de leur circonscription.

7 Actuellement, les jumelages des chambres françaises ou allemandes dépassent souvent le cadre bilatéral ou européen pour se développer avec des chambres situées sur d'autres continents. Ils ont pour principaux objectifs «d'instituer des ponts relationnels avec la compagnie partenaire» et de «créer des partenariats durables». Voir par exemple la politique de la chambre de commerce et d'industrie de Caen sur le site http://www.caen.cci.fr/jumelages-avec-des-cci-etrangeres (consulté le 10 février 2017). 


\section{Un contexte favorable au jumelage}

Dans l'immédiat après-guerre, les chambres de Colmar et de Fribourg-en-Brisgau participent au rétablissement des relations rapides entre institutions consulaires alsaciennes et badoises qui s'opère à l'échelle régionale. Les chambres de commerce du Rhin et de la Moselle sont les principales initiatrices de la reprise des relations commerciales frontalières. Ce volontarisme découle des représentations qu'elles ont de la frontière. Il s'impose également à cause de l'interdépendance des économies frontalières et de la mise en œuvre du Marché commun. Ces trois séries de facteurs expliquent que les chambres alsaciennes œuvrent beaucoup plus rapidement que les acteurs publics à la reprise des relations d'affaires avec le Bade.

Le Rhin d'abord. En 1945, comme en 1918, la plupart des Français le considèrent comme une frontière militaire. Sa largeur, véritable barrière naturelle, est censée interdire toute nouvelle agression allemande. Le contrôler, c'est assurer la sécurité de la France ${ }^{(8)}$. Les militaires français ne disent pas autre chose lorsqu'ils demandent pour la France l'occupation de toute la rive gauche du fleuve et l'installation de têtes de pont sur sa rive droite, visant à faire du Rhin le centre d'une sorte de glacis défensif ${ }^{(9)}$. Pour les chambres de commerce alsaciennes, le Rhin est tout autre. À leurs yeux, il est un axe économique, une voie de transport, un lien propice à des échanges avec l'Allemagne et avec les autres pays riverains. Aussi, dès 1946, les chambres demandent au gouvernement français de constituer une commission interministérielle de l'économie rhénane, qui coordonnerait les efforts en faveur de la navigation sur le $\operatorname{Rhin}^{(10)}$. En parallèle, elles envisagent le développement du port strasbourgeois ${ }^{(11)}$. Bref, dès la fin de la guerre, les chambres veulent faire du Rhin une véritable artère économique de l'Europe occidentale.

L'interdépendance économique des territoires, de part et d'autre de la frontière, est également déterminante dans les prises de position des compagnies alsaciennes. De leur point de vue, le relèvement de l'Alsace dépendra au premier chef de la reprise d'échanges commerciaux avec le Bade, les économies alsacienne et badoise étant dépendantes l'une de l'autre. Cette interdépendance résulte aussi bien de relations nouées de longue date que de l'histoire singulière de l'Alsace, ballotée entre la France et l’Allemagne ${ }^{(12)}$. Par conséquent, nombre d'entreprises alsaciennes ne peuvent trouver

8 Marie Ducet-Huillard, «Le Rhin dans les représentations françaises de 1945 à 1963 », in: Frédéric Dessberg, Frédéric Thébault (dir.), Sécurité européenne. Frontières, glacis et zones d'influence. De l'Europe des alliances à l'Europe des blocs (fin XIX ${ }^{e}$ siècle - milieu XX $X^{e}$ siècle), Rennes, Presses universitaires de Rennes, 2007, p. 129-140, ici p. 130-132.

9 Sur les projets des militaires français relatifs au Rhin, voir les décisions arrêtées par le Comité de défense nationale en août 1944. Ces décisions fixent la doctrine des militaires sur le Rhin pour tout l'après-guerre. «Extrait de la décision prise en séance du Comité de défense nationale ", très secret, État-major de la défense nationale, 12 août 1944, Archives diplomatiques du ministère français des Affaires étrangères, DE-AAA, t. 8. Voir aussi le «mémorandum sur la frontière militaire de l'Ouest européen", État-major de la défense nationale, $1^{\text {re }}$ section, commission d'études de l'armistice, sans date (antérieure au 12 août 1944), Service historique de l'Armée de terre, 4 Q 11 dossier 3.

10 Procès-verbaux des séances de la chambre de commerce et d'industrie (désormais CCI) de Strasbourg, 21 février et 26 septembre 1946, Archives départementales du Bas-Rhin (désormais ADBR), 1430 W 56.

11 Procès-verbal de la séance extraordinaire de la CCI de Strasbourg, 7 octobre 1946, ADBR, 1430 W 56.

12 Bernard Vogler, Histoire politique de l'Alsace de la Révolution à nos jours: un panorama des passions alsaciennes, Strasbourg, La Nuée Bleue, 1995. 
qu'outre-Rhin les pièces de rechange nécessaires à leurs machines. De la même façon, bien des firmes locales se procurent matières premières et produits manufacturés sur la rive allemande du fleuve. Quant aux agriculteurs alsaciens, ils exportent de longue date certaines de leurs productions dans le Bade ${ }^{(13)}$. Entre les compagnies de Colmar et de Fribourg-en-Brisgau d'autres éléments jouent en faveur de la reprise des échanges. La proximité géographique entre les deux instances consulaires est évidemment déterminante. Dans les deux circonscriptions, l'activité industrielle et commerciale respective présente de surcroît de nombreuses similitudes: le secteur textile y est par exemple prépondérant ${ }^{(14)}$.

Mais au sortir de la guerre, la reprise de ces relations d'affaires traditionnelles bute sur la législation en vigueur: les Allemands n'ont pas le droit de commercer avec l'étranger et les échanges entre la France et l'Allemagne, strictement contingentés, sont régis par le seul gouvernement français, qui s'oppose à ce que les industriels et les commerçants interviennent dans les affaires allemandes ${ }^{(15)}$. Pour contourner cette interdiction de principe, les chambres prennent dès novembre 1946, avec l'aval du gouvernement français, l'initiative de créer et de gérer une structure originale, l'Office de compensation des chambres de commerce du Rhin et de la Moselle, dont la tâche essentielle est de permettre la circulation de marchandises et de services qui sont indispensables aux industriels et commerçants alsaciens et mosellans mais qui ne figurent pas dans les plans généraux d'importation et d'exportation français ${ }^{(16)}$. L'accord de clearing ainsi instauré permet la reprise de relations commerciales entre l'Alsace, la Moselle et le territoire de Belfort d'une part, les provinces allemandes de la zone française d'occupation (ZFO) d'autre part, c'est-à-dire le sud du Bade et du Wurtemberg, le Palatinat et la partie méridionale des provinces rhénanes ${ }^{(17)}$. Initialement dirigé par les seules chambres de commerce françaises et à leur seul profit, l'Office se transforme, à partir de l'automne 1948, en un organisme mixte au sein duquel les Allemands, regroupés dans la Kompensationsstelle der Handelskammern, à Fribourg-en-Brisgau, deviennent des partenaires sur un pied d'égalité ${ }^{(18)}$.

En 1949, avec la fin de la période d'occupation stricto sensu, la création de la République fédérale d'Allemagne et la normalisation des relations économiques franco-allemandes ${ }^{(19)}$, l'Office et l'accord de clearing semblent appelés à disparaître. Pour Paris et

13 Patrick J. Schaeffer, L'Alsace et l'Allemagne de 1945 à 1949, Metz, Centre de recherches Relations internationales de l'Université de Metz, 1976, p. 213-223.

14 Procès-verbal de la séance commune de la chambre de commerce et d'industrie de Colmar et de la chambre d'industrie et de commerce de Fribourg-en-Brisgau tenue à Colmar et Fribourg-en-Brisgau le 4 novembre 1959 relative au jumelage des deux compagnies, Archives départementales du HautRhin (désormais ADHR), 2897 W 41.

15 Il s'agit de la loi $n^{\circ} 53$ du SHAEF et de la décision $n^{\circ} 1$ du Conseil de contrôle interallié. Sur la politique économique de la France vis-à-vis de l'Allemagne après 1945, voir Martial LiberA, Un rêve de puissance. La France et le contrôle de l'économie allemande (1942-1949), Bruxelles, P.I.E. Peter Lang, 2012. Procès-verbal de la séance de la CCI de Strasbourg, 14 novembre 1946, ADBR, 1430 W 56. P. J. Schaeffer, L'Alsace et l'Allemagne de 1945 à 1949 (note 13), p. 213-223. Procès-verbal de la séance de la CCI de Strasbourg, 11 mai 1950, ADBR, 1430 W 56. nomiques franco-allemandes de 1945 à 1955. De l'occupation à la coopération, Paris, Comité pour l'histoire économique et financière de la France, 1998. 
Bonn, l'accord commercial franco-allemand, signé en février 1950, rend inutile leur maintien: l'accord commercial prévoit en effet une large libéralisation des échanges franco-allemands qui devrait donner ample satisfaction aux importateurs et exportateurs des deux pays. Mais, cette fois encore, les intérêts des chambres divergent de ceux des gouvernements. Les compagnies craignent que les différences de législation en matière de commerce extérieur entre la France et l'Allemagne tarissent certains courants d'affaires traditionnels et que la gestion d'affaires locales par les administrations au niveau national nuise aux échanges. Aussi, pendant toute l'année 1950, les chambres frontalières ne cessent-elles de faire des propositions à leur gouvernement pour maintenir l'accord frontalier ${ }^{(20)}$. À la fin de l'année, elles obtiennent satisfaction. Un protocole additionnel à l'accord commercial de février 1950 fixe les échanges entre les régions frontalières des deux pays. Il garantit la souplesse des transactions et assure aux chambres un rôle central dans leur détermination. L'accord révise les limites de la zone frontalière au sein de laquelle les transactions sont autorisées. Côté français, elle comprend les départements du Rhin, de la Moselle, et les territoires de Belfort et de la Sarre. Côté allemand, elle recouvre les cercles jouxtant la frontière franco-allemande dans une profondeur d'environ $30 \mathrm{~km}^{(21)}$.

L'entrée en vigueur du Marché commun conduit à son tour les chambres à plus de coopération. La libération progressive des échanges, prévue dans le traité instituant la Communauté économique européenne (CEE), fait perdre à la frontière l'essentiel de ses fonctions de barrière économique. Face à cette nouvelle donne européenne, les organismes économiques des régions frontalières resserrent leur coopération. C'est que, de part et d'autre de la frontière, les problèmes se posent désormais dans les mêmes termes. Pour les chambres, les relations économiques entre l'Alsace et le Bade, les questions d'aménagement du territoire ou de développement des infrastructures de transport doivent être envisagées à l'échelle transfrontalière. Par les modifications que le Marché commun risque d'entraîner dans la structure économique des deux côtés du Rhin, les compagnies de Colmar et de Fribourg-en-Brisgau considèrent nécessaire de renforcer les liens entre les organisations professionnelles représentatives ${ }^{(22)}$. Pour ce faire, contrairement à ce qui s'était passé dans l'immédiat après-guerre, lors duquel ces coopérations avaient été pensées à l'échelle régionale, les structures qui se mettent en place à la fin des années 1950 et au début des années 1960 s'organisent à l'échelon infra-régional et local. Il s'agit de s'entendre avec son voisin direct. Ces structures impliquent souvent différents types d'acteurs, politiques et économiques. La Regio Basiliensis, créée en 1963, en est un exemple ${ }^{(23)}$, la Communauté d'intérêts Moyenne Alsace - Brisgau (CIMAB), instituée la même année entre Colmar et

20 Ibid. Voir aussi les procès-verbaux des séances de la CCI de Strasbourg, 15 juin et 14 décembre 1950, ADBR, 1430 W 56.

21 Procès-verbal de la séance de la CCI de Strasbourg, 8 février 1951, ADBR, 1430 W 57.

22 Résolution adoptée par les chambres de commerce de Colmar et de Fribourg-en-Brisgau réunies en séance plénière commune le 4 novembre 1959 à l'occasion du jumelage des deux compagnies, ADHR, 2897 W 41.

23 Sur la coopération transfrontalière dans l'espace du Rhin supérieur, cf. Birte WAssenberg, Vers une eurorégion? La coopération transfrontalière franco-germano-suisse dans l'espace du Rhin supérieur de 1975 à 2000, Bruxelles, P.I.E. Peter Lang, 2007. 
Fribourg-en-Brisgau en est un autre. Dans ce vaste mouvement, les chambres ne sont pas en reste. Dès le début des années 1960, la CCI de Strasbourg noue par exemple des relations privilégiées avec celle de Stuttgart, son alter ego pour le Bade-Wurtemberg, et avec la compagnie de Lahr, sa voisine directe dans le Bade. Dans les deux cas, les chambres considèrent leurs régions «comme une zone commune de développement au sein de laquelle une collaboration doit s'instaurer dans les différents domaines de l'expansion économique et de l'aménagement du territoire " ${ }^{(24)}$. À Colmar et Fribourgen-Brisgau, les chambres vont plus loin encore dans leur volonté de coopération. En novembre 1959, elles procèdent à leur jumelage.

\section{Un jumelage fécond dans les années $\mathbf{1 9 6 0}$}

Dès le printemps 1959, la compagnie colmarienne profite de l'autorisation qui est accordée en février par l'Assemblée des présidents des chambres de commerce de la Communauté française à une compagnie française de se jumeler avec une institution consulaire étrangère pour faire aboutir le plus rapidement possible le jumelage avec sa sœur fribourgeoise ${ }^{(25)}$. Cette décision, qui s'appuie sur le contexte favorable évoqué précédemment, répond à « un vœu déjà ancien des milieux économiques des deux rives du Rhin en raison des relations étroites de voisinage existant de longue date entre les deux régions voisines. [...] Il s'y ajoute que les deux chambres jouent un rôle identique dans le domaine du commerce extérieur, notamment dans le cadre du régime des échanges frontaliers » ${ }^{(26)}$. L'acte de jumelage, signé à Colmar et à Fribourg le 4 novembre 1959 lors d'une séance commune des deux compagnies, doit beaucoup à l'action des deux secrétaires généraux, Georges Lasch et Adolf Sauter, épaulés par leurs présidents, Robert Schwindenhammer et Hermann Linnemann. Ils en ont été les véritables artisans. Tous les quatre sont nés au tournant du siècle ${ }^{(27)}$. Ils ont vécu les deux guerres mondiales. Dans l'entre-deux-guerres, ils assistent au rétrécissement des relations économiques entre l'Alsace et le Bade. Pendant le second conflit mondial, les deux secrétaires généraux sont victimes de mesures d'éloignement. Celles-ci facilitent leur rapprochement après 1945. Grâce aux contacts établis à titre personnel, les deux hommes en viennent à sympathiser ${ }^{(28)}$. Ils comprennent que l'amélioration des relations entre leurs chambres sera déterminante pour le redressement de leurs économies et plaident dès lors sans relâche pour une coopération renforcée entre leurs institutions.

Dans la pratique le jumelage se traduit, sur le plan formel, par l'organisation annuelle d'une séance commune, alternativement à Colmar et Fribourg. Lors de chacune d'elles,

24 Annexe $\mathrm{n}^{\circ}$ 3: "Visite à la chambre de commerce et d'industrie de Stuttgart» du procès-verbal de l’Assemblée plénière de la CCI de Strasbourg, 11 décembre 1967, ADBR, 1430 W 64.

25 Procès-verbal de la séance commune de la chambre de commerce et d'industrie de Colmar et de la chambre d'industrie et de commerce de Fribourg-en-Brisgau (note 14).

26 Ibid.

27 En 1959, le plus vieux d'entre eux, Hermann Linnemann (né en 1893), a 66 ans. Adolf Sauter, né en 1895, a 64 ans. De leur côté, Georges Lasch et Robert Schwindenhammer, tous les deux nés en 1901, ont 58 ans.

28 Robert Schwindenhammer, «Discours prononcé à l'occasion du jumelage de la chambre de commerce de Colmar et de l'Industrie- und Handelskammer Freiburg im Breisgau», in: IHK Südlicher Oberrhein (éd.), 1959-2009, 50 Jahre Partnerschaft - 50 anniversaire du jumelage IHK Südlicher Oberrhein - CCI Colmar Centre-Alsace, Kehl, Kehler-Druck, 2009, p. 16-17. 
les compagnies traitent ensemble de thèmes variés qui ont une incidence sur leurs activités. En 1959, chacune des chambres présente les caractéristiques de sa circonscription à l'autre. Par la suite, les sujets abordés sont fonction de l'actualité et des dossiers en cours dans les compagnies: moyens de communication, enseignement professionnel, régime des taxes, aménagement du territoire, questions énergétiques, rôle du Rhin dans le développement économique, tourisme et hôtellerie, zones industrielles ou réglementation du commerce de détail. Lors de chacune de ces séances, les membres des deux compagnies présentent la situation dans leur circonscription, leurs projets de développement, les entraves et difficultés de toutes sortes auxquelles elles sont confrontées ${ }^{(29)}$.

Les objectifs des deux chambres sont clairs. Elles entendent dynamiser les relations entre leurs deux circonscriptions. Pour ce faire, elles escomptent un renforcement des moyens de communication - ferroviaire et routier - et un aménagement du territoire qui prennent en compte les évolutions sur l'autre rive du Rhin. Elles tablent également sur une coordination, voire une harmonisation de leur potentiel économique, touristique et culturel. Celle-ci suppose une concurrence non faussée. Sur ce point, les compagnies comptent sur l'action régulatrice de la Commission de la $\mathrm{CEE}^{(30)}$.

Pour l'ensemble de ces objectifs, le jumelage constitue un apport précieux pour les deux chambres. Il leur permet d'abord d'être parfaitement informées de la situation dans l'autre circonscription et des projets de toutes sortes conçus par leur sœur. C'est ainsi que les chambres échangent sur la notion et les structures d'aménagement du territoire qui divergent sensiblement dans leurs deux pays. Les questions de concurrence, en particulier les problèmes liés à sa distorsion, sont également beaucoup analysées. Tout au long des années 1960, les entrepreneurs alsaciens se plaignent en effet de la taxe à l'exportation - Umsatzausgleichsteuer - qu'ils doivent acquitter pour la vente de leurs produits outre-Rhin et dont ils considèrent qu'elle constitue en réalité une mesure permettant à la République fédérale de protéger son marché intérieur. Les compagnies abordent le sujet lors de leur séance commune de 1966 au moment où la Commission de la CEE prévoit de réformer les régimes des taxes sur le chiffre d'affaires - articles 99 et 100 du traité de Rome - dans le but d'éviter que ces derniers ne faussent la concurrence. Paul Schäfer, secrétaire général de la CCI de Fribourg, se félicite que la réforme en cours en Allemagne, qui aboutira au rapprochement du système allemand de celui de la taxe sur la valeur ajoutée (TVA), en vigueur en France depuis 1954, sera plus juste et facilitera les échanges ${ }^{(31)}$. Jusqu'à cette date, le système allemand de taxation est fondé sur une taxe à cascade, c'est-à-dire une taxe cumulative perçue à chaque transaction. Dans le cas d'échanges internationaux, les produits à l'importation en Allemagne sont grevés d'une taxe compensatrice sur le chiffre d'affaires, censée correspondre aux taxes en cascade qui auraient été prélevées si le produit avait été fabriqué en Allemagne. Pour les exportations, les entrepreneurs

29 Sur ce point, voir les procès-verbaux des séances communes entre les deux CCI de 1959 à 1973 dans ADHR, 2897 W 41 à 44.

30 Ibid.

31 «Système et réforme de la taxe sur le chiffre d'affaires en France et en République fédérale d'Allemagne», exposé présenté par Paul Schäfer, secrétaire général de la CCI de Fribourg, lors de la réunion commune des chambres de commerce et d'industrie de Colmar et Fribourg-en-Brisgau, ADHR, 2897 W 42. 
se voient restituer les taxes nationales à la frontière. Schäfer pointe les inconvénients d'un tel système: il contribue à des distorsions sur le plan concurrentiel, la taxe compensatoire ne correspondant pas exactement, selon les produits, à la taxe à cascade, ce qui renchérit les marchandises étrangères sur le marché allemand; ce système fiscal encourage également la concentration industrielle, la diminution des intermédiaires réduisant le prix de revient de production; il est enfin fondé sur un calcul injuste. Le nouveau système, appelé à entrer en vigueur au $1^{\text {er }}$ janvier 1968, sera basé sur une taxe unique qui éliminera les effets cumulatifs de la taxe et rendra possible l'instauration d'une taxe compensatoire équitable dans les opérations de commerce extérieur ${ }^{(32)}$.

Si ces développements sur l'harmonisation des taxes dans les deux pays n'ont qu'une fonction informationnelle, d'autres thèmes étudiés en commun par les chambres les conduisent à se concerter et, dans certains cas au moins, à coordonner leur action dans leur zone mutuelle d'influence, voire même à œuvrer en faveur d'une certaine harmonisation. Les débats sur le tourisme les conduisent par exemple à s'opposer à une logique de concurrence et à promouvoir au contraire un plan concerté qui mise sur les complémentarités entre les sites alsaciens et badois ${ }^{(33)}$.

Le jumelage amène surtout les deux compagnies à se pencher sur des problèmes concrets. Elles constatent par exemple les difficultés de circulation sur le pont reliant Brisach et Neuf-Brisach. Après étude du problème, elles décident d'intervenir auprès des services des travaux publics et de la circulation des deux côtés de la frontière pour que les feux qui règlent la circulation sur le pont à voie alternée restent plus longtemps au vert, permettant ainsi le passage de davantage de véhicules et réduisant de ce fait l'attente pour franchir le pont sur le Rhin ${ }^{(34)}$. Dans le même ordre d'idées, les compagnies sont favorables à l'installation d'un bureau de douane commun à la frontière franco-allemande, grâce auquel les contrôles d'entrée et de sortie seraient facilités et leurs durées considérablement réduites ${ }^{(35)}$.

Au vrai, le partage d'informations entre compagnies présente bien d'autres avantages. Il permet par exemple aux chambres de suivre des dossiers dont la décision dépend des pouvoirs publics des deux États. C'est le cas de la reconstruction du pont ferroviaire entre Colmar et Fribourg. À la fin des années 1950, cette dernière paraît en bonne voie. Lors d'une visite à Colmar en 1959, le général de Gaulle, sensibilisé au projet, demande à Robert Buron, ministre français des Transports, de se saisir du dossier ${ }^{(36)}$. Des échanges ont lieu avec Hans Seebohm, son homologue allemand ${ }^{(37)}$. Des deux côtés du Rhin, on se veut confiant et des assurances sont données. En 1959, le Parlement fédéral prend même une résolution sur la question et appelle le gouvernement allemand à

32 Ibid.

33 Procès-verbal de la séance commune de la chambre de commerce et d'industrie de Colmar et de la chambre d'industrie et de commerce de Fribourg-en-Brisgau (note 14).

34 Séance commune des chambres de commerce et d'industrie de Colmar et de Fribourg-en-Brisgau, 7 décembre 1960, ADHR, 2897 W 41.

35 Ibid.

36 «Rapport sur l'état actuel du projet de reconstruction du pont ferroviaire sur le Rhin», exposé de Georges Lasch lors de la séance commune des chambres de commerce et d'industrie de Colmar et de Fribourg-en-Brisgau (note 34). 
entreprendre avec le gouvernement français toutes les démarches nécessaires en vue de la reconstruction du pont. Grâce aux informations qu'elles se transmettent, les compagnies de Colmar et de Fribourg comprennent que ces prises de contact entre gouvernements n'ont pas véritablement lieu. Elles apprennent de surcroît que le projet bute sur des questions financières, les Allemands insistant pour que la France prenne en charge deux tiers des dépenses, ce que Paris refuse. Par leurs échanges répétés, les compagnies décryptent également la tactique dilatoire du ministère fédéral des Transports et de la Circulation, qui met en avant l'autonomie de décision de la Deutsche Bundesbahn et, par conséquent, la possibilité de cette dernière de s'opposer au projet, pour ne pas faire avancer le dossier ${ }^{(38)}$. Au total, si les deux compagnies ne parviennent pas à peser sur l'issue de la reconstruction du pont, leurs échanges répétés d'informations, conséquence directe du jumelage, leur permettent de pointer les manquements des deux États à leurs engagements.

Grâce au jumelage, l'entraide entre compagnies devient enfin effective. Chacune d'elles peut désormais intercéder auprès de son administration nationale en faveur des ressortissants de l'autre compagnie. Au début des années 1960, la compagnie fribourgeoise saisit par exemple son homologue haut-rhinoise des difficultés que rencontrent les sociétés badoises de transport routier lorsqu'elles sont amenées à circuler en France. Pour chaque voyage, une demande d'autorisation doit être adressée au ministère des Transports à Paris. Comme le traitement de ces demandes est très long, les sociétés allemandes sont contraintes d'envoyer ces dernières des semaines à l'avance, ce qui n'est guère compatible avec la bonne marche de leurs activités. Fait aggravant, il leur faut préciser la nature de leur fret de retour, ce qui est impossible à de si grands intervalles. Bref, les dispositions administratives françaises pèsent sur l'activité des sociétés badoises de transport routier. Or, et c'est là un atout considérable, l'intervention de la CCI de Colmar en faveur des ressortissants de la chambre de Fribourg permet d'obtenir un régime dérogatoire puis l'obtention d'autorisations de circulation désormais valides pour trois mois ${ }^{(39)}$.

À la faveur du jumelage, les ressortissants de chacune des compagnies intéressés par les échanges franco-allemands ont, dans le même ordre d'idées, la possibilité de s'adresser directement à l'autre chambre. Par là même, chacune d'elles est en mesure de renseigner au mieux les ressortissants de l'autre sur la législation en vigueur, la meilleure façon de procéder aux transactions ou les besoins du marché local et régional. Reste que cette véritable «coopération renforcée» entre les compagnies de Colmar et de Fribourg n'est effective que durant les années 1960 et le début de la décennie suivante. À partir de 1973, le jumelage fait les frais de la crise.

\section{Heurs et malheurs du jumelage en temps de crise}

Ses effets sont en effet très différents de part et d'autre du Rhin. Ils creusent les écarts entre les économies alsacienne et badoise et aggravent la dépendance de la première vis-à-vis de la seconde. Cette dissymétrie économique conduit au recul de la

39 Ibid. et séance commune des chambres de commerce et d'industrie de Colmar et de Fribourg-enBrisgau, 4 juillet 1962, ADHR, 2897 W 41. 
coopération transfrontalière au profit de solutions nationales de sortie de crise. À ces enjeux économiques s'ajoute une marginalisation des chambres de commerce dans les structures de coopération transfrontalières et un essoufflement du jumelage entre les compagnies de Colmar et Fribourg-en-Brisgau.

Le long du Rhin, les conséquences de la crise se font sentir avec plus d'intensité en Alsace que dans le Bade. La première est davantage touchée par la désindustrialisation. Pour l'essentiel, cela est dû au fait que l'Alsace comprend plus de branches industrielles fragiles que sa voisine et que, dans bien des cas, l'équipement industriel y a été trop peu modernisé. Les industries de l'habillement, du cuir et du textile sont ainsi massivement touchées. La branche textile, notamment celle localisée dans le Haut-Rhin autour de Mulhouse, connaît de très graves difficultés. Le « déclin industriel ${ }^{(40)}$, pour reprendre l'expression de Bernard Vogler et Michel Hau, touche également les entreprises mécaniques et de la machine-outil. La forte désindustrialisation de l'Alsace par rapport à sa voisine se lit en creux dans l'évolution comparée des taux de chômage. À partir des années 1980, il pèse davantage en Alsace. Les jeunes en sont les principales victimes. Disposant de qualifications professionnelles inférieures à celles de leurs coreligionnaires du Bade, leurs périodes de chômage sont plus longues. Sur le plan du commerce extérieur également, l'Alsace marque divers symptômes d'insuffisance, qu'il s'agisse de la faiblesse de son taux de couverture, de l'étroitesse de son ouverture géographique ou de la fragilité et du manque de rentabilité de ses principaux postes à l'exportation. Au total, les effets dissymétriques de la crise accroissent la dépendance de l'Alsace à l'encontre du Bade. Aux marqueurs traditionnels de cette dépendance - les travailleurs frontaliers alsaciens s'employant en Allemagne et les implantations industrielles allemandes en Alsace, deux phénomènes qui prennent une ampleur considérable à partir des années 1970 - s'ajoutent désormais une certaine emprise foncière et la faiblesse du rayonnement urbain alsacien outre-Rhin qui, dans le cas de Colmar et de Fribourg, se manifeste par un important différentiel de croissance.

Dans ce contexte déprimé, les sorties de crise sont résolument envisagées sur un plan national. L'État français est désormais décidé à prendre en compte les spécificités de l'Alsace en tant que région frontalière et entend la doter d'atouts lui permettant de rivaliser à armes égales avec le Bade. Pour ce faire, les chambres alsaciennes, y compris celle de Colmar, recommandent de dynamiser le commerce extérieur régional en introduisant en Alsace les méthodes commerciales allemandes qui ont fait leur preuve. Un autre point vise à renforcer le tissu industriel alsacien en investissant les espaces en bordure du Rhin. Bref, le glacis défensif d'autrefois doit faire place à des complexes industriels de dernière génération. Les compagnies de Colmar et, indirectement, de Fribourg, sont directement touchées par ces projets. Dans la zone industrielle et portuaire d'Ottmarsheim, les Français prévoient ainsi l'installation d'une aciérie électrique $^{(41)}$. Un peu plus au sud, à Hombourg, une usine d'incinération des déchets est achevée en 1974. C’est le premier établissement de ce type construit en France. Il doit

40 Bernard Vogler, Michel Hau, Histoire économique de l'Alsace. Croissance, crises, innovations: vingt siècles de développement régional, Strasbourg, La Nuée bleue, 1997, p. 347-349.

41 Courrier de la CCI de Mulhouse à la CCI de Fribourg-en-Brisgau a/s du «projet d'implantation d'une aciérie électrique à Ottmarsheim», 22 mars 1974, Wirtschaftsarchiv Baden-Württemberg (désormais WABW), Y 62 Bü 255 H. 
traiter 5000 tonnes de déchets par an ${ }^{(42)}$. En 1978, la centrale nucléaire de Fessenheim commence à produire de l'électricité. À côté de ces usines, les projets de zones industrielles fleurissent. Dans le Haut-Rhin, plusieurs d'entre eux sont en cours de réalisation: à Fessenheim-Nambheim, la zone industrielle, forte de 550 hectares, doit employer 6000 personnes; un peu plus au nord, celle de Neuf-Brisach comprend 650 hectares sur lesquels il est prévu de créer 6000 emplois. À Markolsheim, située à 20 kilomètres de Neuf-Brisach, une autre zone de 800 hectares est en cours d'aménagement $^{(43)}$. Dans le Bas-Rhin, les projets ne manquent pas non plus ${ }^{(44)}$.

Ces initiatives, qui traduisent bien la priorité des solutions nationales dans les schémas français de sortie de crise et d'expansion économique, sont imposées aux Badois sans concertation préalable. Elles conduisent à une crispation des relations sur la frontière, notamment au niveau de Colmar et Fribourg, où un certain émoi saisit les milieux politiques et économiques de la région du Brisgau. Ces derniers appréhendent les effets délétères de ces projets sur leur territoire: accroissement de la pollution fluviale et atmosphérique comme du bruit mais aussi conséquences néfastes sur la qualité de l'habitat et la santé des populations, sur l'image de marque du tourisme local, fondé, grâce au thermalisme et à la Forêt-Noire, sur la nature et la pureté. La chambre de Fribourg réagit donc vivement aux projets français. Elle saisit les ministères compétents du Land et du Bund, d'une part pour les informer, de l'autre pour tenter d'obtenir, par leur entremise, le retrait du projet d'aciérie électrique à Ottmarsheim ${ }^{(45)}$. Elle demande par ailleurs au Verband der chemischen Industrie de l'instruire sur les dangers de pollution que font encourir les usines chimiques travaillant le plomb ${ }^{(46)}$. La compagnie badoise s'enquiert également de la réglementation franco-allemande qui existerait en matière de pollution frontalière. Sur ces questions, de nombreuses instances internationales ont pris position. Le comité des ministres du Conseil de l'Europe a par exemple adopté en 1971 une résolution sur la pollution atmosphérique dans les régions frontalières. Au sein de la CEE, la protection de l'environnement est à l'étude depuis la fin de l'année 1973. Il en va de même pour les membres de l'Organisation de coopération

42 «Aktennotiz für Herrn Dr. Schäfer betr. Industrieansiedlung im Elsaß», chambre de commerce du Südlicher Oberrhein (Fribourg-en-Brisgau), 1 er avril 1974, WABW, Y 62 Bü 255 H.

43 Courrier de Hans Quester et de Paul Schäfer, président et secrétaire général de la CCI du Südlicher Oberrhein, à Günther Hartkopf, secrétaire d'État au ministère fédéral de l'Intérieur, au sujet de la coopération transfrontalière, 3 mai 1974, WABW, Y 62 Bü 255 H.

44 Au sud de Strasbourg, une autre zone est prévue de même qu'à Offendorf, au sud de la raffinerie de Drusenheim. Enfin, une dernière zone industrielle de 200 hectares est en projet à Seltz. Sur tous ces points, voir «Aktennotiz betr. Industrieansiedlung im Elsaß, hier/Gespräche mit der adira und der Kammer Straßburg», chambre de commerce du Südlicher Oberrhein, Ahhy, 2 janvier 1975, WABW, Y 62 Bü 255 H.

45 Courrier du ministère fédéral de l'Économie à la CCI de Fribourg au sujet de «la planification régionale transfrontalière», $1^{\text {er }}$ août 1974; courrier du ministère de l'Économie, des petites et moyennes Entreprises et des Transports du Land de Bade-Wurtemberg à la CCI de Fribourg au sujet de la "coopération transfrontalière dans la région sud du Rhin supérieur», 16 août 1974; courrier du ministère fédéral de l'Aménagement du Territoire, de la Construction et de l'Urbanisme à la CCI de Fribourg au sujet de la «coopération franco-allemande dans le domaine de l'aménagement du territoire », 27 août 1974, WABW, Y 62 Bü 255 H.

46 Courrier du Verband der chemischen Industrie à la CCI de Fribourg, 2 janvier 1975, WABW, Y 62 Bü $255 \mathrm{H}$. 
et de développement économique qui prévoient de proposer en novembre 1974 une recommandation sur la question de la pollution transfrontalière. Mais, quelles que soient ces dispositions internationales, force est de constater qu'aucune réglementation franco-allemande n'existe ${ }^{(47)}$. À Fribourg, Paul Schäfer, le secrétaire général de la CCI, prend acte de la relative inefficacité de la coopération transfrontalière dans certains domaines, en particulier pour les questions d'aménagement du territoire. À la fin de l'année 1974, son constat est amer. La coopération transfrontalière lui semble illusoire parce que les lois et réglementations sont pensées et destinées à s'appliquer dans un cadre strictement national qui ne prend nullement en compte la dimension transnationale de la coopération aux frontières. Quant aux autorités nationales, elles sont responsables devant leurs administrés mais en aucun cas devant ceux du pays voisin. Leur poids dans les hiérarchies politiques et administratives conduit en outre à faire valider prioritairement chaque projet par les pouvoirs publics centraux, les instances frontalières n'étant saisies qu'en cas d'accord sur le plan national. Bref, derrière la coopération affichée et portée haut, Paul Schäfer regrette le maintien de pratiques, notamment administratives, centrées sur le seul espace national ${ }^{(48)}$. Sans doute n'est-il pas loin de partager l'avis du Spiegel, qui consacre au printemps 1977 un article à la coopération transfrontalière au titre provocateur: «Seule la pollution est transfrontalière ${ }^{(49)}$.

Pour Schäfer, il est vain d'essayer de continuer à réduire les barrières que constituent les lois nationales. Cette voie lui semble sans issue. De son point de vue, la solution réside bien davantage dans une dynamique constructive, telle la création d'une commission binationale chargée des questions d'aménagement du territoire. Il lui paraît donc essentiel d'obtenir que les instances de coopération frontalière, qui sont en 1974 et 1975 en pleine phase d'institutionnalisation, comprennent des structures réfléchissant à l'aménagement du territoire frontalier au sens large et que les compagnies consulaires soient représentées en leur sein ${ }^{(50)}$. Ses espoirs, partagés par les chambres alsaciennes et badoises, sont déçus. Le traité de Bonn, signé le 3 novembre 1975, qui institutionnalise la coopération transfrontalière sur le Rhin supérieur entre la France, la République fédérale d'Allemagne et la Suisse, qui définit les compétences des organismes décisionnels et en arrête la composition ${ }^{(51)}$, écarte en effet les CCI. Combiné

47 Karl Moersch, ministre d'État au ministère des Affaires étrangères, au président du Parlement allemand, au sujet de la "coordination dans le Rhin supérieur lors de l'instauration de grands projets", 13 novembre 1974, WABW, Y 62 Bü 255 H.

48 Courrier de Paul Schäfer, secrétaire général de la CCI de Fribourg, à Fr. Dole, président de la CCI de Cologne au sujet de la coopération transfrontalière, 23 décembre 1974, WABW, Y 62 Bü 255 H.

49 «Grenzüberschreitend ist nur der Schmutz», Der Spiegel, n² 20, 9 mai 1977, WABW, Y 62 Bü 255 H.

50 Courrier de Paul Schäfer, secrétaire général de la CCI de Fribourg, à Fr. Dole, président de la CCI de Cologne au sujet de la coopération transfrontalière (note 48 ).

51 Elles se composent d'une commission intergouvernementale et de deux comités régionaux. L'un est dévolu à la coopération franco-allemande dans la partie Nord du Rhin supérieur, l'autre à la coopération trinationale autour de Bâle, Mulhouse et Lörrach. Sur ce sujet, cf. Birte WAssEnBERG, «Die Entwicklungsgeschichte der grenzüberschreitende Zusammenarbeit am Oberrhein seit 1963», in: Anita Prettenthaler-Ziegerhofer, Michael Kissener, Jan Kisber (dir.), Zwischenräume. Grenznahe Beziehungen in Europa seit den 1970er Jahren, Innsbruck/Vienne/Bolzano, Studienverlag, 2011, p. $25-48$, ici p. 31. 
aux difficultés économiques, ce recul de l'influence consulaire dans les dossiers de coopération transfrontalière conduit les compagnies de Colmar et de Fribourg-enBrisgau à désinvestir leur jumelage. Il faut dire qu'en la matière la pérennisation des relations entre les deux compagnies s'est routinisée. Au fil du temps, l'élan initial, redevable à l'engagement et au volontarisme des secrétaires généraux, bute sur les conséquences de la crise et s'essouffle pendant la décennie 1970. Pourtant, à partir des années 1980, le jumelage connaît un second souffle. Sur le plan spatial, il s'élargit en 1981 à l'ensemble des chambres de commerce alsaciennes. Du côté allemand, le champ de la coopération est étendu aux chambres de Fribourg et de Lahr qui, depuis 1973, ont fusionné pour former la chambre du Südlicher Oberrhein. De nouvelles missions, qui s'inscrivent dans le renforcement des relations directes entre entreprises, sont alors confiées aux compagnies frontalières. Il leur revient de mener en commun des travaux d'analyse conjoncturelle et structurelle des économies alsacienne et badoise ${ }^{(52)}$, d'organiser des bourses de produits ou de dynamiser les exportations communes de petites et moyennes entreprises (PME) innovantes des deux rives du Rhin. Avec la relance européenne du milieu des années 1980, les compagnies participent également à un programme de la Commission européenne qui vise à dynamiser les transferts de technologie entre $\mathrm{PME}^{(53)}$.

$\mathrm{Au}$ total, le jumelage entre les chambres de Colmar et de Fribourg-en-Brisgau est riche d'enseignements. Il confirme premièrement le rôle essentiel des forces profondes, des éléments structurants sur le long terme, comme le Marché commun, qui poussent au rapprochement entre les compagnies. Il souligne également l'importance des initiatives prises par les acteurs consulaires. Reste que ces facteurs déterminants peuvent avoir des effets strictement inverses: que l'interdépendance des économies frontalières soit remise en cause ou que les relations entre acteurs se distendent et l'étroitesse des relations entre compagnies est alors immédiatement revue à la baisse. Deuxièmement, entre la fin des années 1950 et le début des années 1980, les finalités du jumelage évoluent considérablement. Pensé au départ pour faciliter les relations entre acteurs économiques d'un pays et pouvoirs publics de l'autre, le jumelage devient progressivement, avec la relance européenne et la libéralisation des échanges, un intermédiaire entre acteurs économiques des deux circonscriptions et un relais des institutions européennes. Il faut troisièmement rappeler la précocité de ce jumelage entre compagnies consulaires. Il précède les rapprochements au niveau politique et en constitue, par les milieux économiques, administratifs et politiques qu'ils touchent, un préalable indispensable.

52 «Réunion commune des chambres de commerce et d'industrie d'Alsace et du Bade-Wurtemberg: projet d'allocution du président Moritz», chambre régionale de commerce et d'industrie d'Alsace, service des études, 3 décembre 1981, WABW, Y 60 Bü 1278.

53 Sur toutes ces questions, voir Martial Libera, Diplomatie patronale aux frontières. Les relations des chambres de commerce frontalières françaises avec leurs homologues allemandes (1945 - milieu des années 1980), Genève, Librairie Droz, 2017 (à paraître au second semestre). 


\section{Résumé}

Cet article étudie le jumelage économique institué depuis 1959 entre les chambres de commerce et d'industrie de Colmar et de Fribourg-en-Brisgau. Il retrace d'abord sa genèse et souligne que des éléments structurants sur la frontière - signature du Marché commun; échanges économiques franco-allemands soutenus - ont favorisé le rapprochement entre compagnies. Il présente ensuite son fonctionnement dans les années 1960, analyse les domaines dans lesquels il conduit à une coopération approfondie entre chambres et montre qu'il a contribué au renforcement des relations transnationales entre institutions consulaires. Il relate enfin les difficultés auxquelles le jumelage est confronté à partir de 1973. À ce propos, il rappelle que les chambres ont été exclues de l'institutionnalisation de la coopération transfrontalière en 1975 alors que leur travail en commun avait constitué un préalable indispensable à ces initiatives. 